\title{
Multi-Objective Evolutionary Based Feature Selection Supported By Distributed Multi-Label Classification And Deep Learning On Image/Video Data
}

\author{
Gizem Nur Karagoz \\ G.Karagoz@tudelft.nl \\ Delft University of Technology \\ Delft, Netherlands
}

\begin{abstract}
We live in an era in which a myriad of computer systems produce immense amounts of (raw) data every day. This big data must be processed efficiently to gain valuable and hidden knowledge. Complex processing pipelines need to be designed for filtering out irrelevant data, also for efficient data mining and machine learning methods must be used to discover useful correlations in the big data. The purpose of this $\mathrm{PhD}$ research is the implementation of multi-objective evolutionary-based dimensionality reduction on a high volume of image/video data with the support of distributed multi-label classification algorithms.
\end{abstract}

\section{CCS CONCEPTS}

- Computing methodologies $\rightarrow$ Distributed artificial intelligence; Feature selection.

\section{KEYWORDS}

distributed machine learning, big data processing, feature extraction, dimensionality reduction, feature engineering

\section{ACM Reference Format:}

Gizem Nur Karagoz. 2021. Multi-Objective Evolutionary Based Feature Selection Supported By Distributed Multi-Label Classification And Deep Learning On Image/Video Data. In 22nd International Middleware Conference Doctoral Symposium (Middleware '21 Doctoral Symposium), December 6-10, 2021, Virtual Event, Canada. ACM, New York, NY, USA, 2 pages. https: //doi.org/10.1145/3491087.3493675

\section{INTRODUCTION}

In the emerge of the enormous amount of image/video data; social media surveillance cameras, wearable devices, smartphones and more various other resources take role day by day. For example, on one of the most popular social media platforms, YouTube, 144.000 hours of video were generated per day in 2019, and this number has increased to 720.000 hours of video in 2021. On the other hand, numerous CCTV cameras are placed in cities all over the world. For instance, in London, 422.000 CCTV cameras are recording Terabytes of videos every day, and the number of sources is expected to increase in time. [6] The size of the raw data obtained from these

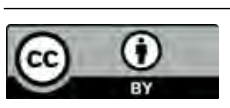

This work is licensed under a Creative Commons Attribution International 4.0 License. Middleware '21 Doctoral Symposium, December 6-10, 2021, Virtual Event, Canada (C) 2021 Copyright held by the owner/author(s).

ACM ISBN 978-1-4503-9155-9/21/12.

https://doi.org/10.1145/3491087.3493675
Table 1: Feature Selection(FS) Results conducted by Autoencoder(AE) and Genetic Algorithm(GA)

\begin{tabular}{lllllll}
\hline \multirow{2}{*}{$\begin{array}{l}\text { Base } \\
\text { Classifier }\end{array}$} & \multicolumn{2}{l}{ Before FS } & \multicolumn{2}{l}{ After GA } & \multicolumn{2}{c}{ After AE } \\
\cline { 2 - 7 } & HS & $\begin{array}{l}\text { \# of } \\
\text { Feat }\end{array}$ & HS & $\begin{array}{l}\text { \# of } \\
\text { Feat }\end{array}$ & HS & $\begin{array}{l}\text { \# of } \\
\text { Feat }\end{array}$ \\
\hline J48 & 0.8383 & & 0.886 & 5 & 0.8486 & 10 \\
SVM & 0.8511 & 42 & 0.886 & 5 & 0.8532 & 6 \\
LR & 0.8431 & & 0.8653 & 6 & 0.8433 & 2 \\
\hline
\end{tabular}

video sources goes beyond being processable with traditional solutions. The collection of large data cannot be beneficial unless it is being processed. Having sufficient solutions over these collections only possible with an efficient data pre-procecssing which provides to reveal correlations in the big data efficiently. Therefore, both applying big data processing solutions and data pre-processing operations are required in the first stage of handling an enormous amount of data. Eliminating noisy or redundant information provides remarkable improvements for both faster solutions and more accurate results. Especially for image and video data, there might be hundreds of features that are extracted from a single frame, but only a few features are related to the target object. At this point, feature selection is becoming an important part of image classification over local feature descriptors and even over deep features. The core part and the motivation of this $\mathrm{PhD}$ research is implementation of a middleware for multi-objective dimensionality reduction on multi-label image/video data. Thus, a high volume of data will be able to be handled and also, classification accuracy will be increased while the number of features is decreased.

\section{RELATED WORK}

In a previous study [7], multi-objective feature selection is applied on MIR-Flickr image dataset. A selection of the most relevant results are represented in Table 1. After the feature selection is applied with the genetic algorithm the hamming-score(HS), which indicates the average accuracy of multi-label classification, is increased from 0.83 to 0.88 while the number of features(\# of Feat) is decreased from 42 to 5 . This proves feature selection on image features is essential to get better results since the unrelated information is eliminated. In this case, one of the most challenging parts of handling image/video data is the 'volume' of the data [2]. Especially while extracting image feature descriptors, loading the data completely into the memory could not be possible with a single computer for eight million videos, for instance. Thus, a scalable middleware comprising of a distributed file system and co-located processing capabilities will be required for the solution of the storage and availability of the 
data. Multi-objective optimization is applied for feature selection before in the literature. However, most of the studies cover video processing and feature selection separately[3], [4]. An application of feature selection on big image/video data is not addressed before to the best of our knowledge. The novelty of this research is the improvement of the classification performance for big image/video data through optimized features in a multi-objective manner.

\section{RESEARCH APPROACH}

This $\mathrm{PhD}$ research is focused on dimensionality reduction with multi-objective evolutionary algorithms on big image/video data in a distributed manner. The main research question is:

- How can we implement distributed multi-label machine learning algorithms to improve the performance of the multiobjective evolutionary algorithm for feature selection on multi-label image/video data?

We plan to address this overarching question by looking into the concrete challenges of;

- How should the feature extraction process be improved through parallelization or in a distributed manner?

- Is it possible to boost the performance of state-of-the-art multi-objective evolutionary algorithms by distributed implementation?

- How to affect the other state-of-the-art multi-objective evolutionary algorithms in both objective manners as the number of features and classification accuracy?

- What are the efficient distributed multi-label classification algorithms for different domains?

First, the subset selection of multi-objective evolutionary algorithm will be parallelized since one of the most efficient tools for optimization of NP-Hard problems is parallel multi-objective evolutionary algorithms [5]. Optimization performance directly depends on chromosome calculation for big data and sufficient number of iterations which turns this process into an inapplicable situation. Therefore, keeping a population at the master processor's memory and sharing chromosomes with workers for the fitness calculation will be the answer for the core of this research. Handling such enormous amount of data storage and processing requires a distributed solution as it is not small enough to be stored on a single machine. A big data solution is one of the best option to store/process distributed data. Within this scope, the research stages;

Feature Extraction will be the first stage of processing the data. Raw data is the input of this stage, and local feature descriptors or deep features of images/frames should be extracted from previously separated frames. All these steps require a big data solution because of the data volume. Extracting the bag of visual words and creation of codebook for only 1000 images took ten hours in previous work [7], and many more images would be required in the current research for more accurate results, improvement on the classification performance and the reliability of extracted features.

Dimensionality Reduction happens in the second stage. After local feature descriptors are extracted,this stage will be applied to eliminate unnecessary features and get more valuable data. For this purpose, parallel implementation of NSGA-II and one or two more state-of-the-art multi-objective evolutionary algorithms (i.e. Particle Swarm Optimization(PSO)) will be implemented.
Evaluation is the third and last phase of the implementation. Fitness calculation is provided while the feature selection operation is performing. However, final features should be evaluated eventually. For this phase, distributed multi-label classification algorithms will be implemented. Validation of the proposed model will be performed on a large scale video/image dataset. For this purpose the Youtube-8M dataset, which is the largest multi-label video classification dataset, will be used [1]. Additionally, some smaller-scale surveillance audio-visual datasets is a convenient alternative for the comparison of different domained problems. After all the results are obtained, an analysis should be carried out with a proper data visualization approach. Results should be well defined to show the important points that are revealed within the scope of research.

\section{CONCLUSION}

This $\mathrm{PhD}$ research aims to develop distributed machine learning algorithms for dimensionality reduction on the high volume of multilabel image/video data. Dimensionality reduction will be applied with multi-objective evolutionary algorithms. The performance of the feature selection is planned to be improved by considering both the accuracy of results and the execution time of the evolution phase with the implementation of distributed multi-label classification algorithms. Additionally, a big data solution is provided with all required modules of big data architecture by addressing some core big data characteristics such as volume, variety, and value. Detailed comparison and discussions with the other state-of-theart multi-objective evolutionary algorithms would be a remarkable contribution to the literature of this research. Additionally, since we expect feature extraction to become an important part of processing pipelines for future applications, we are aiming at solving this problem in the form of a reusable middleware. This newly created audio-visual dataset and middleware for feature extraction will be publicly available as the additional contribution of this research.

\section{ACKNOWLEDGMENTS}

This $\mathrm{PhD}$ research is supervised by Jan Rellermeyer and Dick Epema at TU Delft in the Distributed Systems Group.

\section{REFERENCES}

[1] Sami Abu-El-Haija, Nisarg Kothari, Joonseok Lee, Paul Natsev, George Toderici, Balakrishnan Varadarajan, and Sudheendra Vijayanarasimhan. 2016. Youtube-8m: A large-scale video classification benchmark. arXiv preprint arXiv:1609.08675 (2016).

[2] Kiran Adnan and Rehan Akbar. 2019. An analytical study of information extraction from unstructured and multidimensional big data. fournal of Big Data 6, 1 (2019), $1-38$.

[3] Aftab Alam, Irfan Ullah, and Young-Koo Lee. 2020. Video Big Data Analytics in the Cloud: A Reference Architecture, Survey, Opportunities, and Open Research Issues. IEEE Access 8 (2020), 152377-152422.

[4] Noura AlNuaimi, Mohammad Mehedy Masud, Mohamed Adel Serhani, and Nazar Zaki. 2020. Streaming feature selection algorithms for big data: A survey. Applied Computing and Informatics (2020).

[5] Erick Cantú-Paz et al. 1998. A survey of parallel genetic algorithms. Calculateurs paralleles, reseaux et systems repartis 10, 2 (1998), 141-171.

[6] Boqing Gong, Wei-Lun Chao, Kristen Grauman, and Fei Sha. 2014. Diverse sequential subset selection for supervised video summarization. Advances in neural information processing systems 27 (2014), 2069-2077.

[7] Gizem Nur Karagoz, Adnan Yazici, Tansel Dokeroglu, and Ahmet Cosar. 2020. A new framework of multi-objective evolutionary algorithms for feature selection and multi-label classification of video data. International fournal of Machine Learning and Cybernetics (2020). https://doi.org/10.1007/s13042-020-01156-w 